\title{
A Novel Calibration Method for Object Plane Tilt Angle Measurement in Microscope System
}

\author{
CUI Li ${ }^{1, a}, Y U M i^{1, b}$ \\ ${ }^{1}$ Ningbo University, Zhejiang 315211 \\ Faculty of Information Science and Engineering, NBU Ningbo, China \\ aEmail: cuili_mail@126.com, ${ }^{\text {b} E m a i l: ~ y u m e i 2 @ 126 . c o m ~}$
}

Keywords:Microscopes; Camera calibration; Rotating disks; Flexible structures; Imaging geometry

\begin{abstract}
A novel calibration method is proposed to measure object plane tilt angle in microscope system with small-depth-field. The proposed method is constructed by a space model about calibration board and the surface of image. Only a rotating disk, a goniometer and a calibration board with some features are needed, the tilt angle between the object plane and image plane and their geometric relationship can be estimated by the space model. Compared with some classical calibration methods with expensive equipment, the proposed method is easy to be implemented with relative cheap equipments. Moreover, it has been proved that the proposed method is quite efficient through experimental verification. It can also contribute to three dimensional applications in computer vision of microsystems.
\end{abstract}

\section{Introduction}

With the rapid development of microscopic technologies, precise measurement and high accuracy machining had stepped into the process of industrial applications, such as steel for hot working, assembly of precision electronic components or precision instruments, lens calibration, and so on [1, 2]. However, due to the small-depth-field of optical microscope amplification characteristic, the precise observation is greatly restricted under microscope. Such as defocusing caused by object plane tilt in microscope system may influence the assembly of auxiliary measuring device. In terms of precise medical observation, people adjust working distance to observe object multi-facetedly by rotating object stage of microscope system. However, it is usually inefficient and dissatisfactory in the industrial precision instrument operation. Hence, object plane and image plane in the microscope system should be precisely calibrated by extracting and matching features in image accurately. The camera calibration operation is very important for subsequent dealing with tiny objects such as precise measurement, calibration, three dimensional (3D) reconstruction [3].

Calibration problem had been studied by Hekilla [4], Tsai [5], Zhang [6] etc., and some conclusions had been made, for example monocular convex lens plain surface calibration [7], and it is believed that with three directional rotations, almost all internal and external parameters of camera can be calibrated with chessboard calibration. However, for digital optic microscope, Tsai's method does not consider the nonlinear distortion of lens and must get the scale factor, so that its accuracy may not be guaranteed. Zhang's method has to limit the object plane rotation angle from 15 to 45 degree. The unsatisfactory results also exist in Ravn's method. Due to the limitation on small-depthfield and small field of view in optical microscope system, the precision of the above calibration methods will be more or less influenced [8]. In the existing cognitive, there is a paradox, the images easy to be matched may normally be hard to be calibrated; on the other hand, the abundant characteristics of calibration source normally cannot be applied to be matched directly. No matter in the optimization of photogrammetry [9], or mold measuring to build better optical platform [10], there are such a phenomenon.

For high precision 3D reconstruction after matching, tiny deviation is often fatal. The difficulty in calibration is how to find the exact matching sets for data analysis. Some matching algorithms had been proposed [11, 12]. In this paper, on the basis of exact matching, with the spatial geometric relationships, a tilt angle measuring method is proposed for object plane rectification in microscope system. 


\section{Object plane tilt in optical stereoscopic microscope}

This paper aims to such a test platform with optical stereoscopic microscope that the object is with a small-depth-field, which can be seen from previous works [13]. The experimental platform uses a stereo microscope of ZOOM460N, as shown in Fig. 1(a). In the figure, "A" is a dual camera with independent light path, " $\mathrm{B}$ " is the rotating disk with the rotatory accuracy of 1 degree manual adjusted, and "C" is the angle control platform. The lights of observed object first go through a large lens, then go into the two separate light path, go across the objective lens, and then the lights are captured on both sides of the relative camera. The used cameras are 902B Watec of Japanese Company, directly connect the interface to the eyepiece of stereomicroscope. Each frame is obtained by the camera with the size of $704 \times 576$. Besides, the accuracy of angle control system is 0.0025 degrees with the precision stepper motor adjusted. That is to say each of 400 steps makes angle control system rotate at one degree. Test sets are divided into 5 degree values, which can be adjusted from 0 to 4 degrees.



Figure 1. Experimental platform

According to the properties of microscope system, the camera imaging model can be got. However, the object plane tilt exists more or less, but in microscope system, parallelity between image plane and object plane is significant to the subsequent processing. Hence, it is necessary to estimate the angle between the image plane and object plane.

This paper intends to get the correct angle between the image plane and object plane, so as to solve the problem of local blurring caused by angular deviation of the two planes.

\section{New calibration method for measuring object plane tilt angle}

In most cases, camera calibration can be described by using radial and tangential distortion model, and the object surface can be fitted by different calibration methods. It is known that calibration error and accuracy of calibration model have a great influence on subsequent matching and 3D reconstruction. Based on good matching of feature points for plane rectification, the proposed method can be used in monocular as well as binocular microscope system. Moreover, monocular rectification can be used to deduce binocular polar plane rectification.

\section{A. Geometric model building}

In this paper, a rotating disk is used to simulate the actual situation. The rotating disk can provide a feature constraint, through which the feature of points and finally the tilted object plane can be got. The calibration model is usually based on the following two accepted assumptions [14]:

- The model is a pin-hole model which allows us to consider the focus point as a real mathematical coordinate point;

- The acquisition system is reliable.

Fig. 2 represents the relationships between the image plane and object plane. Theory is if the object is at a distance, one can assume the light rays will arrive perpendicularly to the plane of the lens, and thus converge at the focal point. In Fig. 2, the upper part denotes the image plane, while the lower part is the object plane which may tilt in microscope system (presuppose that object is as a particle in object plane). Fig. 2(a) shows the ideal relationship between the image plane and object plane, in which the two planes are parallel with each other. The imaging model in Fig. 2(b) is the actual slant imaging, which is used to calculate the tilt angle of the two planes. By calculating the 
relative relationship between the object plane and the image plane, the relative distortion coefficients can be got, and finally the object plane is able to be rectified to the ideal plane model in Fig. 2(a).
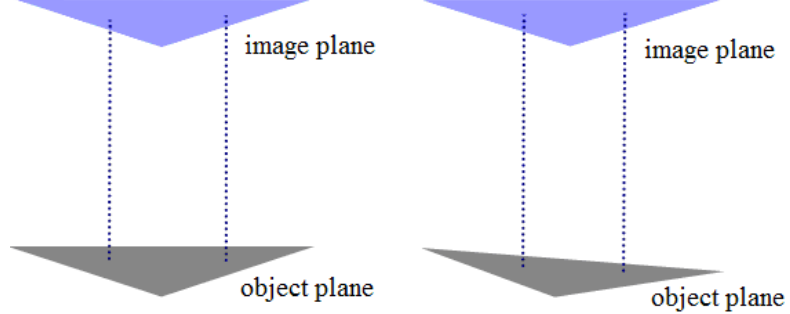

(a) Ideal model (b) Actual model

Figure 2. Relationship between image and object planes

In view of projection between image plane and object plane, a new calibration method for measuring object plane tilt angle is proposed and described as follows:

- Select two image feature points named as $\mathrm{p} 0 \mathrm{i}$ and $\mathrm{p} 0 \mathrm{j}$ according to the projection of linear fitting (here according to the actual situation more feature points can be selected);

- Rotate the rotating disk with an angle $\alpha$;

- Extract the two image feature points $\mathrm{p} 1 \mathrm{i}$, and $\mathrm{p} 1 \mathrm{j}$ corresponding to $\mathrm{p} 0 \mathrm{i}$ and $\mathrm{p} 0 \mathrm{j}$ after rotating, and compute the rotation angle $\theta$ in image plane;

- Calculate the relationship between object plane and the image plane according to formulas deduced in the follows.

\section{B. Model caculating}

On the basis of the experimental platform, space model can be drawn in Fig. 3(a). In the figure, plane ABF denotes the actual tilted object plane $\Gamma_{\mathrm{r}}$, while plane DBE $\Gamma_{\mathrm{p}}$ is the translated image plane so that the image plane and object plane have the same center of rotation and easy to be analyzed. Plane $\mathrm{ABC} \Gamma_{\mathrm{a}}$ is a auxiliary plane, $\mathrm{AC}$ is parallel to $\mathrm{DE}$ and perpendicular to FE. In Fig. 3(a), $\angle A B F=\alpha$ denotes the actual rotatory angle, $\angle A B C=\beta$ denotes the space auxiliary rotary angle. $\angle D B E=\theta$ denote the image rotary angle, which can be obtained from the captured images. $A B=B F=B C$ is the actual rotary radius. Do auxiliary vertical $F H \perp B E, D G \perp B E, A X \perp B C, D H$ is Euclidean distance of the feature points rotating in the image, which can be measured from the captured images. Let $\rho$ denote the intersection angle of the object planar $\Gamma_{\mathrm{r}}$ and the image plane $\Gamma_{\mathrm{p}}$, $R$ be the rotary radius of image. By midperpendicular theorem, the center of rotation in image can be obtained. Fig. 3(b) shows an image combined with images before and after rotating. In Fig. 3(b), the points at 3 and 5 scale marks are two pairs of the selected feature points, that is, the pairs $\left(p_{0}{ }^{i}\right.$, $\left.p_{1}{ }^{i}\right)$ and $\left(p_{0}{ }^{j}, p_{1}{ }^{j}\right)$. From a pair of feature points before and after rotation, the midpoint of the corresponding feature points can be determined, so that the slope of the midperpendicular of the pair of feature points can be calculated. Further, the intersection of the two midperpendiculars corresponding to the two pairs of feature points which is actually the center of rotation in image can be obtained. As shown in Fig. 3(b), the blue lines are the connecting lines between the center of rotation and the corresponding feature points. Then the image rotary angle $\theta$ can be calculated. Besides, the rotary radius of image $R$ can also be calculated, and the actual rotary radius can further be computed with the resolution ratio of the images.

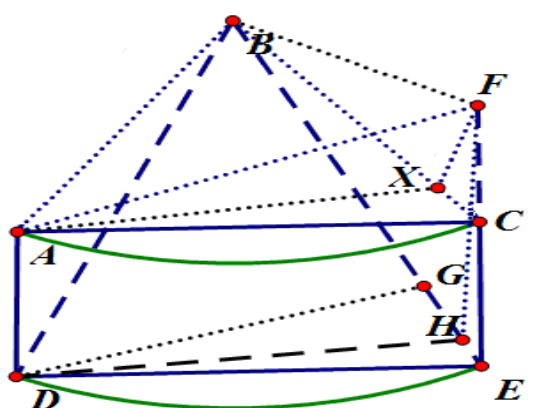

(a)

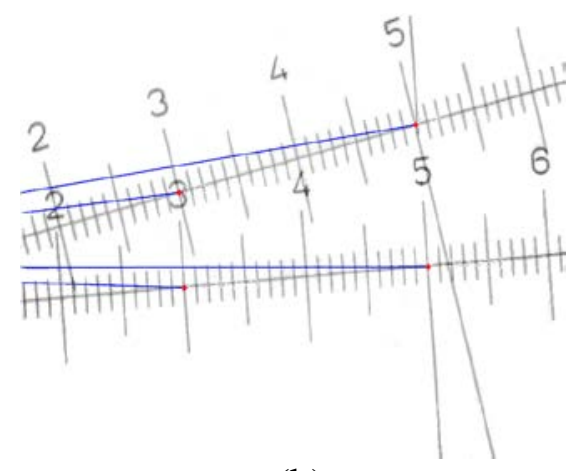

(b)

Figure 3. The geometry model 
According to tetrahedron volume formula, and $S_{B E F}=0.5 \cdot F H \cdot B E$ and $S_{B D E}=0.5 \cdot B E \cdot D G$, we can get as in (1)

$$
V_{\mathrm{DBFE}}=\frac{1}{3} D G \cdot S_{\triangle B E F}=\frac{1}{3} F H \cdot S_{\triangle B D E}
$$

Thus $\Gamma_{\mathrm{BEF}} \perp \Gamma_{\mathrm{p}}$, and the space hypothesis shown in Fig. 3(a) is proved.

Let $\angle A B D=\angle C B E=\eta$ be the plane shaft angle, and $\angle F B C=\gamma$ represent the axis angle between auxiliary plane and actual tilted object plane, then the relationship of space angle can be built, and the space relationship of surface space structure can be constructed with all the feature points in the rotary images. When the rotation angle is an acute angle, according to three cosine theorem or the Pythagorean Theorem of $\triangle A F X$, there exists

$$
\cos \alpha=\cos \beta \cdot \cos \gamma
$$

And since $D E=A C$, we can get

$$
2 \cos \eta \sin \frac{\theta}{\overline{\overline{2}}} 2 \sin \frac{\beta}{2}
$$

By the above equations, the relationship between the angels $\beta$ and $\eta$ can be got, substituting (2) into (3), (4) is deduced

$$
\frac{\cos \alpha}{\cos \gamma}=\left(1-\cos ^{2} \eta(1-\cos \theta)\right)
$$

And there is $B H=R \cdot \cos (\eta+\gamma)$, according to the law of cosines, there is

which can also be expressed as

$$
\cos \theta=\frac{\mathrm{DB}^{2}+B H^{2}-D H^{2}}{2 D B \cdot B H}
$$

$$
\begin{aligned}
& 2 R^{2} \cos \eta \cos (\eta+\gamma) \cos \theta \\
& =R^{2} \cos ^{2} \eta+R^{2} \cos ^{2}(\eta+\gamma)-D H^{2}
\end{aligned}
$$

Moreover, according to the Pythagorean Theorem $D G^{2}+G H^{2}=D H^{2}, A X^{2}+X F^{2}=A F^{2}$, there are

$$
\left\{\begin{array}{l}
(\cos \eta \sin \theta)^{2}+(\cos (\eta+\gamma)-\cos \eta \cos \theta)^{2}=D H^{2} \\
\sin ^{2}(\beta)+\sin ^{2} \gamma=4 \sin ^{2}(\alpha / 2)
\end{array}\right.
$$

where $D H$ is Euclidean distance of the feature points rotating in the image. From (2) to (7), the approximate closed interval solutions $\beta, \gamma$ and $\eta$ can be obtained by a high order univariate polynomial equation with Newton iteration method. According to the rule of triangle available distance, we can get

$$
\left\{\begin{array}{l}
F H=R \cdot \sin (\eta+\gamma) \\
A D=R \cdot \sin \eta
\end{array}\right.
$$

In the process of derivation, there is a very important prior knowledge that the image plane DBE $\Gamma_{\mathrm{p}}$ and auxiliary plane ABC $\Gamma_{\mathrm{a}}$ are all perpendicular to the surface BEF $\Gamma_{\perp}$. Although the above derivation process is tedious, the operation is relatively simple by using Cosine Formula and the Pythagorean Theorem.

From the above equations, the space auxiliary rotation angle $\beta$, the first rotatory angle $\eta$, the second rotatory angle $\gamma$ between two planes and the rotation radius of $R$ and $\rho$ can be got.

According to the above calculative results, a space rectangular coordinate system can be set. Regard the center of rotation $\mathrm{B}$ as the original point. $B D$ as the positive direction of $x$, normal vector of plane $\Gamma_{\mathrm{p}}$ can be set as $n_{p}=(0,0,1)$. Normal vector of plane $\Gamma_{\mathrm{r}}$ can be calculated by points $\mathrm{A}, \mathrm{B}, \mathrm{F}$

According to the Cross-measurement,

$$
\left\{\begin{array}{l}
A:(\mathrm{R} \cos \eta, 0, R \sin \eta) \\
B:(0,0,0) \\
\mathrm{F}:\left(\mathrm{F}_{x}, F_{y}, F_{z}\right) \\
\mathrm{F}_{\mathrm{x}}=\mathrm{R} \cos \theta \cos (\eta+\gamma) \\
F_{y}=\mathrm{R} \sin \theta \cos (\eta+\gamma) \\
F_{z}=\mathrm{R} \sin (\eta+\gamma)
\end{array}\right.
$$




$$
\left\{\begin{array}{l}
\mathrm{n}_{r}=\left(n_{r x}, n_{r y}, n_{r z}\right) \\
n_{r x}=-\tan \eta \\
n_{r y}=\tan \eta \cot \theta-\frac{\tan (\eta+\gamma)}{\sin \theta} \\
n_{r z}=1
\end{array}\right.
$$

The normal angle between two planes can be obtained (when $\rho$ is acute angle)

$$
\rho=\left\{\begin{array}{l}
\pi-\arccos \left(\frac{n_{r} \cdot n_{p}}{\left|n_{r}\right| \cdot\left|n_{p}\right|}\right) \\
\arccos \left(\frac{n_{r} \cdot n_{p}}{\left|n_{r}\right| \cdot\left|n_{p}\right|}\right)
\end{array}\right.
$$

As mentioned above, the related parameters can be calculated by the formulas derived.

\section{Experimental results}

In order to obtain the deviation angle between image plane and the object plane, an experiment of nearly horizontal rotation and vertical fine-tuning has implemented to verify the derived geometric formulas and to get the angular deviation of the two planes in the rotation. Based on the experiment platform shown in Fig. 1, a calibration board is first placed on the rotating disk, focused within the depth of field, and then captured to get the images. After that, horizontally adjust the rotating disk with a rotary angle $\alpha$, capture the calibration board again. Later, vertically adjust the rotating disk with a rotary angle $\varphi$, and repeat the above steps to take a group of scale plate rotary images and fixed angle rotary images, so as to verify the effectiveness of the proposed method for estimating the angular deviation of the two planes. During the process, rotating disk and angle control platform are firstly adjusted to nearly horizontal plane, so as to simulate the ideal model of the image plane and object plane, and to get some initial parameters such as image resolution and magnification of the microscope system.

Table 1 shows the parameters calculated with the selected calibration feature points when horizontal rotary angle $\alpha=10^{\circ}$ and step motor rotary angle $\varphi=0^{\circ}, 1^{\circ}, 2^{\circ}, 3^{\circ}, 4^{\circ}$, respectively. The case $\varphi=0^{\circ}$ is used to simulate the ideal model shown in Fig. 2. In the table, the unit of length is pixel, while the unit of angle is degree. $S_{r}$ is normalized equivalent radius scale $\left(S_{r}=S_{0} / S_{i}\right.$, where $S_{i}$ is radius scale corresponding to the $i$ th row of the table), $S_{a}$ is the angle control platform rotatory scale by the step motor $\left(S_{a}=\alpha / \theta\right)$.

According to Table 1, the average rotary radius of image $R$ after rotation, denoted as $\mu_{R}=\frac{1}{N} \sum_{\mathrm{i}=1}^{N=4} R_{i}$, equals to 1174.47 , and the deviation of $R$, denoted as $D_{\mathrm{R}}=\sum_{\mathrm{i}=1}^{N=4}\left(R_{i}-\mu_{R}\right)^{2}$, is 1.16033 . According to the gradual change of $\rho$ within the scope of depth in preliminary measurements, initial inherent deviation $\Delta \rho=0.27936$ can be obtained. The relationship among $\rho$, rectified $\rho$ and $\varphi$ is shown in Fig. 4 . From Fig. 4, it is seen that the distribution of $\rho$ and $\varphi$ is nearly linear, which is consistent to the hypothesis indicated by the deduced formulas. But there are still some deviations, it is mainly resulted from the artificial matching feature points and the limitation of image resolution.

In the proposed method, if the slope of midperpendicular of a pair of feature points is nearly infinity, it will affect the calculation results and robustness of the proposed method. In such a case, the feature points have to be changed. 


TABLE I. CALCULATED PARAMETERS IN THE EXPERIMENTS $(\alpha=$
\begin{tabular}{|l|l|l|l|l|l|}
\hline \multirow{2}{*}{$\boldsymbol{\varphi}$} & \multicolumn{5}{c|}{ Parameters } \\
\cline { 2 - 6 } & $\boldsymbol{\eta}$ & $\boldsymbol{\gamma}$ & $\boldsymbol{\rho}$ & $\boldsymbol{R}$ & rectified $\boldsymbol{\rho}$ \\
\hline $0^{\circ}$ & 0 & 0 & 0 & 1149.64 & 0 \\
\hline $1^{\circ}$ & 1.08692 & 1.30479 & 0.7205 & 1172.71 & 0.99986 \\
\hline $2^{\circ}$ & 1.18743 & 3.08260 & 1.7001 & 1176.65 & 1.97946 \\
\hline $3^{\circ}$ & 2.18743 & 5.07950 & 2.7861 & 1172.53 & 3.06546 \\
\hline $4^{\circ}$ & 6.32553 & 5.72969 & 3.3965 & 1175.99 & 3.67586 \\
\hline
\end{tabular}

There are several facts that influence the measurement accuracy of the proposed method, including 1) image resolution; 2) the rotary angle is too small to get a small statistical data variance; 3) unavoidably, uneven phenomenon occurs in the process of measurement to planar points. Therefore, the measurement can be implemented repeatedly so as to get more accurate statistical results.

In the experiments, it is found that when $\rho$ is within 3 degrees the sharp area of image is larger than that the angle larger than $3^{\circ}$. The measured initial inherent deviation $\Delta \rho$ can be used to compensate so that better results can be achieved.

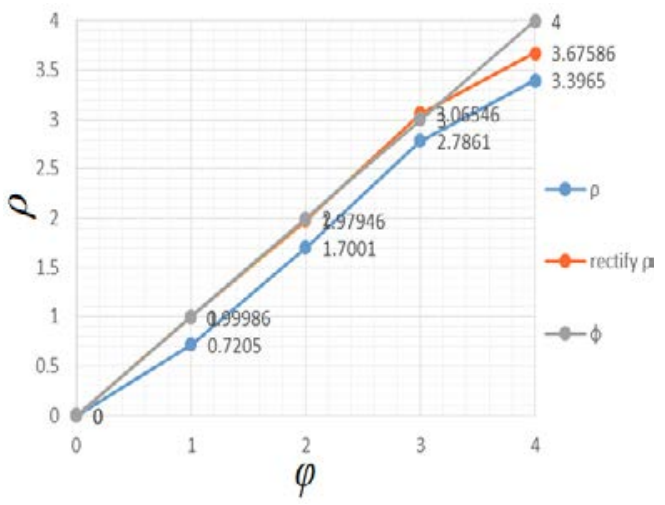

Figure 4. The relationship among $\rho, \varphi$ and rectified $\rho$

\section{Conclusion}

Due to the limitation of small-depth-field and small field of view by using microscope, a new method is proposed to cope with the defocusing caused by object plane tilt. According to the proposed method for the object plane angle measurement, the object plane can be rectified. Monocular rectification can be deduced to binocular polar plane rectification, so the proposed method can be used in monocular as well as binocular microscopes. Generally, in real applications, it needs to know the flatness of object plane, so the proposed method can be used widely, for example, calibrating the observation platform, further for 3D surface reconstruction, measurement, etc. The proposed method is simple and effective, well suited to be used in situation without much specialized knowledge of three dimensional geometry or computer vision. For the problem of Cartesian coordinate slope, local polar coordinates processing analysis may get better results. For the uncertainty of matching points, using better matching algorithm to get sub-pixel matching feature points, may achieve better results. In addition, with binocular depth and corresponding matching algorithm, the proposed method can be further deduced to reconstruct three dimensional curved surface, which can be applied to industrial camera with fixed assignments.

\section{Acknowledgement}

This work is supported by the Natural Science Foundation of China (Grant Nos. 61271270, 61311140262). 


\section{References}

[1] S. Dutta, S. K. Pal, S. Mukhopadhyay, R. Sen, “Application of digital image processing in tool condition monitoring: A review”. CIRP, vol. 6, 2013, pp. 212-232, doi:10.1016/j.cirpj.2013.02.005

[2] D Ji, R. Yang, L. Zhang, B. Wang, X. Chen, "The research of medical microscopic image mosaic based on the algorithm of surf." IEEE Conference on ICCWAMTIP, 2013, pp. 16-20, doi: 10.1109/ICCWAMTIP.2013.6716591

[3] C. Nguyen, D. Lovell, R. Oberprieler, D. Jennings, M. Adcock. E, GatesStuart and JL. Salle, "Virtual 3D Models of Insects for Accelerated Quarantine Control”. IEEE International Conference on ICCVW, 2013, pp. 161 167, doi: 10.1109/ICCVW.2013.27

[4] J. Heikkila, O. Silvén, “A four-step camera calibration procedure with implicit image correction”, proc. IEEE Conference on Computer Vision and Pattern Recognition, 1997, pp. 11061112, doi: 10.1109/CVPR.1997.609468

[5] RY. Tsai, “A versatile camera calibration technique for high-accuracy 3D machine vision metrology using off-the-shelf TV cameras and lenses", IEEE Journal of Robotics and Automation, vol. 3, 1987, pp. 323-344, doi: 10.1109/JRA.1987.1087109

[6] Z. Zhang, “A flexible new technique for camera calibration”, IEEE Transactions on Pattern Analysis and Machine Intelligence, vol. 22, 2000, pp. 1330-1334, doi: 10.1109/34.888718

[7] M. Ammi, V. Frémont, A. Ferreira, "Automatic camera-based microscope calibration for a telemicromanipulation system using a virtual pattern”, IEEE Transactions on Robotics, vol. 25, 2009, pp. 184-191, doi: 10.1109/TRO.2008.2006866

[8] F.Z. Fanga, X.D. Zhanga, et al., "Manufacturing and measurement of freeform optics”, vol. 62, 2013, pp. 823-846, doi:10.1016/j.cirp.2013.05.003

[9] A. Gallo, M. Maurizio, and B. Fabio, “3D reconstruction of small sized objects from a sequence of multi-focused images”, Journal of Cultural Heritage, vol. 15, Mar.-Apr. 2014, pp. 173182, doi:10.1016/j.culher.2013.04.009

[10]D. Ryu, B. Dong, T. Davidson, A. Burton, "Model-based micro profile measurement using multi-focused images for micro gear assembly", IEEE International Conference on Micro Engineered and Molecular Systems (NEMS), 2010, pp. 844-849. doi: 10.1109/NEMS.2010.5592210

[11]Q. Yang, “A non-local cost aggregation method for stereo matching”, IEEE Conference on Computer Vision and Pattern Recognition (CVPR) 2012, pp. 1402-1409, doi: 10.1109/CVPR.2012.6247827

[12]X. Mei, X. Sun, W. Dong, H. Wang and X. Zhang, "Segment-Tree based Cost Aggregation for Stereo Matching”. IEEE Conf. on Computer Vision and Pattern Recognition 2013, pp. 313-320, doi: 10.1109/CVPR.2013.47

[13]S. Fan, M. Yu, Y. Wang, G. Jiang, “A depth estimation method based on geometric transformation for stereo light microscope” Bio-Medical Materials and Engineering, vol. 24, 2014, pp. 2743-2749, doi:10.3233/BME-141092.

[14]B. Peuchot, “Camera virtual equivalent model 0.01 pixel detectors”, Computerized medical imaging and graphics, vol. 14, 1993, pp. 289-294, doi:10.1016/0895-6111(93)90019-J 